\title{
Adverse drug reaction monitoring during antimi- crobial therapy for septicemia patients at a univer- sity hospital in New Delhi
}

\author{
Muhammad Shamshir Alam ${ }^{1,2}$, Krishna Kolappa Pillai ${ }^{1}$, Syed Aliul Hasan Abdi ${ }^{1}$, Prem Kapur ${ }^{3}$, \\ Paru Kutty Pillai ${ }^{4}$, and Kandasamy Nagarajan ${ }^{5}$
}

\begin{abstract}
${ }^{1}$ Department of Pharmacy Practice and Clinical Pharmacy, Unaizah College of Pharmacy, Qassim University, Unaizah, Kingdom of Saudi Arabia; ${ }^{2}$ Department of Pharmacology, Faculty of Pharmacy, Hamdard University, New Delhi; ${ }^{3}$ Department of Medicine, Hamdard Institute of Medical Sciences \& Research and Hakeem Abdul Hameed Centenary Hospital, Hamdard University, New Delhi; ${ }^{4}$ Department of Microbiology, Majeedia Hospital, Hamdard University, New Delhi; ${ }^{5}$ Department of Pharmaceutical Chemistry, KIET School of Pharmacy, Delhi, India
\end{abstract}

Received: December 23, 2015 Revised : December 30, 2016 Accepted: January 21, 2017

\section{Correspondence to}

Muhammad Shamshir Alam, Ph.D. Department of Pharmacy Practice and Clinical Pharmacy, Unaizah College of Pharmacy, Qassim University, Unaizah, Kingdom of Saudi Arabia

Tel: +91-9650286178

Fax: $+966-63800662$

E-mail: shamshir_pharma@yahoo.com
Background/Aims: Adverse drug reaction (ADR) is an appreciably harmful or unpleasant reaction, resulting from an intervention related to the use of a medicinal product. The present study was conducted in order to monitor the frequency and severity of ADR during antimicrobial therapy of septicemia.

Methods: A prospective, observational, and noncomparative study was conducted over a period of 6 months on patients of septicemia admitted at a university hospital. Naranjo algorithm scale was used for causality assessment. Severity assessment was done by Hartwig severity scale.

Results: ADRs in selected hospitalized patients of septicemia was found to be in $26.5 \%$ of the study population. During the study period, 12 ADRs were confirmed occurring in 9, out of 34 admitted patients. Pediatric patients experienced maximum ADRs, $44.4 \%$. Females experienced a significantly higher incidence of ADRs, $66.7 \%$. According to Naranjo's probability scale, $8.3 \%$ of ADRs were found to be definite, $58.3 \%$ as probable, and $33.3 \%$ as possible. A higher proportion of these ADRs, $66.7 \%$ were preventable in nature. Severity assessment showed that more than half of ADRs were moderate. Teicoplanin was found to be the commonest antimicrobial agent associated with ADRs, followed by gemifloxacin and ofloxacin.

Conclusions: The incidence and severity of ADRs observed in the present study was substantially high indicating the need of extra vigilant during the antimicrobial therapy of septicemia.

Keywords: Sepsis; Antimicrobial therapy; Anti-bacterial agents

\section{INTRODUCTION}

Presence of evidence of infection together with systemic responses like fever, hypothermia, tachycardia, etc. is described as sepsis [1]. Sepsis has alternately been called septicemia [2] or sepsis syndrome [3]. Septicemia is a common cause of pediatric morbidity and mortali- ty [4]. World Health Organization (WHO) estimates that $85 \%$ of newborn deaths are due to infections, including sepsis, pneumonia, and tetanus [5]. Forty percent of the infants identified with sepsis die, and the biggest toll is in developing countries [5]. Considering the possible severe outcome and paucity of time, administration of empirical antimicrobial therapy is justified in patients 
suspected of septicemia. Appropriate empiric antibiotic therapy is associated with enhanced survival as well as a shortened hospital stay [6].

Safety and tolerability of pharmaceuticals have always been an interest and concern for those who receive and prescribe drugs. Antimicrobial agents are no exception, and with their great benefit to achieve, there must be responsibility of clinicians and consumers to understand their risks. Regardless of the antimicrobial to be used, a risk-benefit analysis should be used to weigh the risks of toxicity and antibiotic resistance against the intended therapeutic benefit. According to WHO, "an adverse drug reaction (ADR) is any response to a drug which is noxious and unintended and occurs at doses normally used in man for prophylaxis, diagnosis or therapy of disease or the modification of physiological function." This definition excludes therapeutic failures, intentional and accidental poisoning, and drug abuse [7]. ADRs not only may result in hospital admission or prolonged hospitalization but also may lead to permanent disability or even death. Drug reactions can be classified into immunologic and nonimmunologic etiologies. The majority ( $75 \%$ to $80 \%$ ) of ADRs are caused by predictable, nonimmunologic effects [8]. Remaining $20 \%$ to $25 \%$ of adverse drug events are caused by unpredictable effects that may or may not be immune mediated [8]. Predictable ADRs may either be avoidable or tolerated, implying such events as toxicity, drug interactions, and secondary effects. Unpredictable and usually unavoidable ADRs include idiosyncratic or allergic reactions as well as intolerance.

Monitoring and documentation of ADRs including preventable and nonpreventable ADRs are crucial to encourage and to ensure safe use of pharmacologic agents. For assessing ADRs, other causes for the adverse drug events should be excluded; a temporal relationship between drug use and onset of the event should exist; improvement should be noted following drug cessation, reactivation upon rechallenge of the drug should be noted; and the reaction is known to be associated with the drug in question. The exact incidence of specific adverse events is often impossible to assess, although clues are gathered from multiple sources. Awareness of the risk groups may decrease the incidence of preventable and serous antimicrobial related adverse effects as well as the extra cost incurred due to these untoward effects.
Previously we have showed the antimicrobial therapy and outcome of septicemia patients [9]; therefore, the current study was conducted to monitor the incidence and assess severity of ADRs during the antimicrobial therapy of septicemia patients admitted at a university hospital.

\section{METHODS}

\section{Study design and population}

The present study was a prospective, observational, and noncomparative one that was conducted at Majeedia Hospital (now, Hakeem Abdul Hameed Centenary Hospital), a 150-bed teaching hospital located in Hamdard University Campus, New Delhi, India. The study population included 34 clinically diagnosed septicemia patients who were admitted in Majeedia Hospital during the period from July 2009 to December 2009.

\section{Patient selection}

The research protocol was approved by Jamia Hamdard Institutional Review Board (Approval Letter No. 07/09, JH-IRB dated July 2009). Written informed consent was obtained from the patients or patients' close relative prior to inclusion in the study. Fig. 1 describes flow chart of the study patients. After a thorough scrutiny of 186

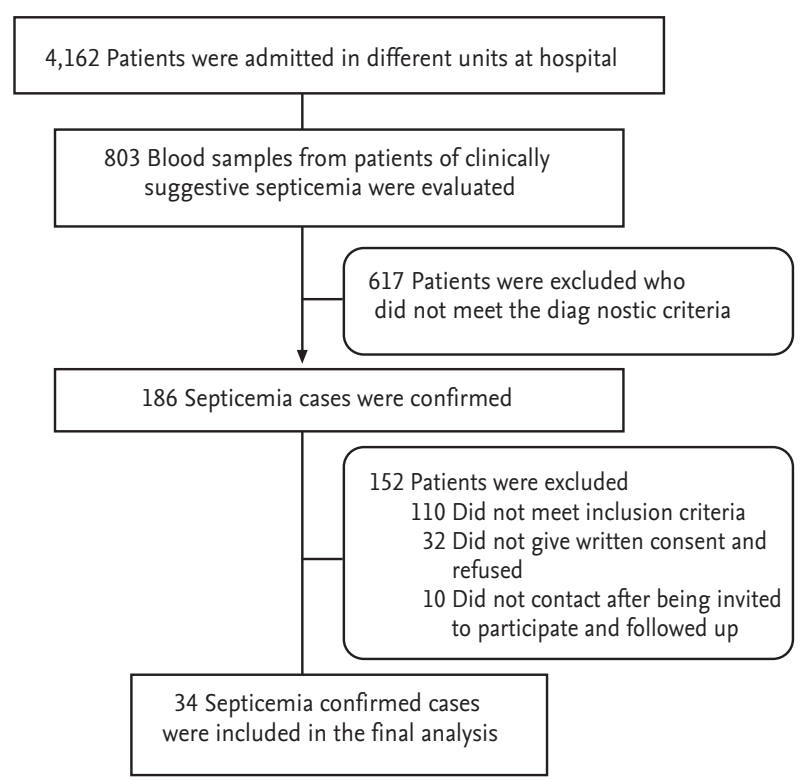

Figure 1. Flow chart for the selection of study patient. 
septicemia cases, 34 patients were selected on the basis of inclusion and exclusion criteria. Patients of either sex irrespective of age with suspected or proven infection who fulfill the diagnosing criteria and were prescribed at least one antimicrobial agent were included in the present study. Patients with intentional, accidental poisoning, alcoholic and drug abuse were excluded from the study.

\section{Diagnostic criteria for septicemia}

The clinical diagnosis of septicemia was made by the physician after recording the clinical symptoms followed by performing the standard laboratory tests in the laboratory of Majeedia Hospital. Septicemia diagnoses were done based on with suspected or proven infection and two of the following six criteria [10]: (1) fever (oral temperature $>38^{\circ} \mathrm{C}\left[101^{\circ} \mathrm{F}\right]$ or hypothermia $<$ $36^{\circ} \mathrm{C}\left[96.8^{\circ} \mathrm{F}\right]$ ); (2) tachypnoea (> 24 breaths/min or arterial $\mathrm{pCO}_{2}$ below $4.25 \mathrm{kPa}$ ); (3) tachycardia (heart rate $>90$ beats/min), leukocytosis $(>12,000 / \mu \mathrm{L})$ or leukopenia (< $4,000 / \mu \mathrm{L}$ ) or $>10 \%$ bands; (4) systolic blood pressure < $90 \mathrm{mmHg}$ or mean arterial pressure $<65 \mathrm{mmHg}$; and (5) altered mental state or oliguria or thrombocytopenia.

\section{Data collection procedure}

All relevant data including patient age, gender, all drugs, the patients received prior to the onset the reactions, their respective dosage, route of administration, frequency of administration, date and time of onset of reaction, and patient's allergy status to drugs and food were recorded. If any drug induced reactions occurred, the physicians were informed and discussed about the possible antimicrobial related ADRs. The patients were assessed for the possible reaction and carefully monitored on daily basis during their hospital stay and for their occurrence on the basis of specific structured questionnaires. Anatomical Therapeutic Chemical (ATC) code was assigned to each drug for their identification. When offending drug was not known, timing and order of dechallenge/rechallenge were decided by the treating physicians. The decisions made by the physicians were treated as final decision. Other relevant details were recorded from patients' medication records or their treatment chart.

The estimation of the probability that a drug caused an adverse clinical event is usually based on clinical judgment. Using the conventional categories and definitions of definite, probable, possible, and doubtful ADRs generates wide variability in assessment of the exact nature of ADR. Different scales categorize the causality relationship in different ways. For example, the WHO scale categorizes the causality relationship into certain, probable, possible, unassessable/unclassifiable, unlikely, and conditional/unclassified. Naranjo et al. [11] developed a simple method to assess the causality of ADRs in a variety of clinical situations, and its systematic application to different cases of alleged ADRs has provided reliable answers. According to Naranjo scale, the probability that the adverse event is related to drug therapy is expressed as definite, probable, possible, or doubtful, which are based on simple questionnaire that can be answered rapidly [11]. The Naranjo's ADR probability scale was used during the study to estimate the probability of ADRs. Hartwig preventability and severity scales were used to measure the preventable and nonpreventable nature of ADRs and also to assess severity of the ADRs [12].

\section{RESULTS}

\section{Patient demographics}

Out of 186 septicemia cases admitted in hospital wards, 34 patients were selected on the basis of inclusion and exclusion criteria. There were 22 males $(64.7 \%)$ and 12 females (35.3\%). The patients were in the age range between neonates, nine (26.5\%); 1 month to 14 years, 10 (29.4\%); 14 to 59 years, 10 (29.4\%); and 60 years and above, five $(14.7 \%)$.

\section{ADRs during antimicrobial therapy of septicemia}

In the present study, the detection of ADRs in selected hospitalized patients of septicemia were found to be in $26.5 \%$ of the study population. Details of ADRs, their classification, interventions, and outcomes are summarized in Table 1. During the study period, 12 ADRs were confirmed occurring in nine, out of 34 admitted patients. Most of these ADRs, 10 (83.3\%) were detected by the attending physicians, whereas two ADRs (16.7\%) were detected by clinical pharmacist. Majority of patients experienced at least one ADR, while few of the patients reported more than one during the study period. One pa- 
Table 1. Analysis of ADRs against different parameters

\begin{tabular}{|c|c|}
\hline Parameter & Number (\%) \\
\hline \multicolumn{2}{|l|}{ Age } \\
\hline Neonate & o \\
\hline 1 mon-14 yr & $4 / 9(44 \cdot 4)$ \\
\hline $14-59 \mathrm{yr}$ & $3 / 9(33.3)$ \\
\hline$\geq 60 \mathrm{yr}$ & $2 / 9(22.2)$ \\
\hline \multicolumn{2}{|l|}{ Sex } \\
\hline Male & $3 / 9(33 \cdot 3)$ \\
\hline Female & $6 / 9(66.7)$ \\
\hline \multicolumn{2}{|l|}{ Onset of ADRs } \\
\hline Acute $(<1 \mathrm{hr})$ & 0 \\
\hline Sub-acute (1-24 hr) & $10 / 12(83.3)$ \\
\hline Latent (> 2 day) & $2 / 12(16.7)$ \\
\hline Unknown & o \\
\hline \multicolumn{2}{|l|}{ Causality } \\
\hline Definite & $1 / 12(8.3)$ \\
\hline Probable & $7 / 12(58.3)$ \\
\hline Possible & $4 / 12(33 \cdot 3)$ \\
\hline \multicolumn{2}{|l|}{ Severity } \\
\hline Mild & $4 / 12(33 \cdot 3)$ \\
\hline Moderate & $7 / 12(58.3)$ \\
\hline Severe & $1 / 12(8.3)$ \\
\hline \multicolumn{2}{|l|}{ Nature of ADRs } \\
\hline Preventable & $8 / 12(66.7)$ \\
\hline Nonpreventable & $4 / 12(33 \cdot 3)$ \\
\hline \multicolumn{2}{|l|}{ Intervention } \\
\hline Stop the medication & $5 / 12(41.7)$ \\
\hline Reduce the dose & $2 / 12(16.7)$ \\
\hline Add another drug & o \\
\hline Substitute another drug & $3 / 12(25.0)$ \\
\hline No change & $2 / 12(16.7)$ \\
\hline \multicolumn{2}{|l|}{ Outcome } \\
\hline Fully recovered & $8 / 12(66.7)$ \\
\hline Recovering & $3 / 12(25.0)$ \\
\hline Fatal & o \\
\hline Unknown & $1 / 12(8.3)$ \\
\hline
\end{tabular}

ADR, adverse drug reaction.

tient on an average reported at least 1.3 ADRs. Pediatric patients (1 months to 14 years) experienced maximum ADRs ( $n=4,44.4 \%)$. Females experienced a significantly higher incidence of ADRs ( $n=6,66.7 \%$ ) than males $(\mathrm{n}=3,33.3 \%)$. A large proportion of the ADRs, $10(83.3 \%)$ were sub-acute type, appeared within 1 to 24 hours of the therapy and two (16.7\%) were latent type, appeared after 2 days of the therapy.

According to Naranjo's probability scale, 8.3\% ADRs were found to be definite, $58.3 \%$ as probable and $33.3 \%$ as possible. A higher proportion of these ADRs, eight (66.7\%) were preventable in nature, whereas four (33.3\%) were nonpreventable. Severity assessment showed that the more than half of ADRs were moderate (58.3\%), mild (33.3\%), and severe (8.3\%). In $41.7 \%$ of the observed ADRs cases, the offending agents were stopped. In 25\% ADRs management antimicrobials was substituted with other agents. Dose reduction was needed in only one patient (16.7\%). No change in treat-ment was attempted in one patient (16.7\%) who experienced ADRs. In majority of the patients $(66.7 \%)$, complete recovery was achieved. There were no fatal outcomes observed due to ADRs. The most common antimicrobial agents implicated with ADRs and their reaction details were presented in Table 2. Teicoplanin (one prescription) was found to be the commonest antimicrobial agent associated with ADRs (n $=3,300 \%)$, followed by gemifloxacin $(\mathrm{n}=2,100 \%)$ and ofloxacin ( $\mathrm{n}=2,40 \%)$. Among organs gastrointestinal tract (50\%) was the most frequently affected organ system with ADRs, while skin (33.3\%) was the second most organ system affected with ADRs (Table 3). Relation of ADRs with sex, age and comorbid conditions in patients of septicemia has been shown in Table 4 .

\section{DISCUSSION}

The present study illustrates the magnitude of the problem of ADRs in selected hospitalized patients of septicemia with detection of ADRs in $26.5 \%$ of the study population. This report is higher than the findings of as other study [13] and comparable to a joint study report of United States and Canada where the incidences ranged from $15 \%$ to $27 \%$ [14]. Differences in methodology for data collection (e.g., spontaneous reporting versus chart review) may explain the variability in reported frequencies.

In the present study, higher numbers of ADRs were observed in female patients as compared to male patients (Table 1). Hence, females developed ADRs 3.6 times more often than males. It might be attributed that the females being more sensitive to the effect of drugs 
Table 2. Antimicrobials most commonly involved in ADRs and their reaction details

\begin{tabular}{lclcc}
\hline Antimicrobial & No. of prescriptions & \multicolumn{1}{c}{ Reaction detail } & ADRs $(\mathrm{n}=12)$ & ATC code \\
\hline Amoxicillin-clavulanic acid & 15 & GTCS (1), blood with stools $(1)$ & $2 / 15(13 \cdot 3)^{\mathrm{a}}$ & Jo1CRo2 \\
Ceftriaxone-tazobactam & 8 & Diarrhea $(3)$ & $3 / 8(37.5)^{\mathrm{a}}$ & Jo1CGo2 \\
Ofloxacin & 5 & Itching (1), popular rashes $(1)$ & $2 / 5(40)^{\mathrm{a}}$ & Jo1MAo1 \\
Gemifloxacin & 2 & Skin rash (1), loose motion $(1)$ & $2 / 2(100.0)^{\mathrm{a}}$ & Jo1MA15 \\
Teicoplanin & 1 & Skin rash (1), vomiting (1), ND (1) & $3 / 1(300)^{\mathrm{a}}$ & Jo1XAo2 \\
\hline
\end{tabular}

ADR, adverse drug reaction; ATC, Anatomical Therapeutic Chemical; GTCS, generalized tonic clonic seizure; ND, nasal decongestion.

a(\%) Values are presented as number of ADR/number of prescription.

Table 3. Organ systems affected due to ADRs

\begin{tabular}{lc}
\hline Organ system & No. $(\%)$ of ADRs $(\mathrm{n}=12)$ \\
\hline Gastrointestinal & $6(50.0)$ \\
Skin & $4(33.3)$ \\
Central nervous system & $1(8.3)$ \\
Nose & $1(8.3)$ \\
\hline
\end{tabular}

ADR, adverse drug reaction.

Table 4. Relation of ADRs with sex, age, and comorbid conditions in patients of septicemia

\begin{tabular}{lcc}
\hline Characteristic & No. of patients & $\begin{array}{r}\text { Patients with } \\
\text { ADRs, } \mathrm{n}(\%)\end{array}$ \\
\hline Sex & 22 & $3(13.6)$ \\
$\quad$ Male & 12 & $6(50.0)$ \\
$\quad$ Female & & \\
Age, yr & 29 & $7(24.1)$ \\
$<60$ & 5 & $2(40.0)$ \\
$\geq 60$ & & $1(14.3)$ \\
Comorbid condition & 7 & $8(29.6)$ \\
\hline Less than 2 conditions & 27 & \\
More than 2 conditions & &
\end{tabular}

ADR, adverse drug reaction.

than the males [15-17]. Incidence of ADR was 1.6 times higher in patients, who were aged more than 60 years of age (Table 4). Subsequently incidence of ADR was two times higher in patients with more than two comorbid conditions. It has been observed that there is correlation between number of ADRs and the number of comorbid conditions in the patients.
A case of generalized tonic clonic seizure associated with amoxicillin-clavulanic acid (Augmentin, GlaxoSmithKline, London, UK; on dose 250:50 mg frequency four times daily route of administration intramuscular administration) was observed in the present study. This might be linked to $\beta$-lactam antibiotics in which $\beta$-lactam ring is present, which directly or indirectly can stimulate central nervous system. Blood in stool, $1 / 15$ (6.7\%) reactions associated with amoxicillin-clavulanic acid was observed. A study from Italy reported $2 \%$ hematological reactions associated with amoxicillin-clavulanic acid [18]. It may be linked due to thrombocytopenia and prolongation of prothrombin time, which are described as the uncommon side effects in the summary of product characteristics of amoxicillin-clavulanic acid [19]. However, we found little information about hematological reactions related to amoxicillin-clavulanic acid.

The higher incidence of ceftriaxone-tazobactam-induced diarrhoea could be explained by its greater resistance to $\beta$-lactamases, which might result in a higher concentration of ceftriaxone in the intestine and a consequent stronger modification of the bacterial flora, responsible for diarrhoea. Although these effects are usually minor and transient, they may lead to the interruption of treatment; therefore, further studies are necessary to assess the role of ceftriaxone in gastrointestinal disturbances.

Ofloxacin induced itching and purpural rashes were observed in one patient out of five prescription drug (20\%). Liang et al. [20] reported $2 \%$ of skin rash as an ADR due to ofloxacin in a comparative study of ofloxacin versus co-trimoxazole for prevention of infection in neutropenic patients. Gemifloxacin induced skin rash 
was detected in one patient out of two prescriptions, $50 \%$ patients. It also caused diarrhea in one patient out of two prescriptions. A study reported appearance of rash more common with use of gemifloxacin, particularly in younger women ( 39 years old) treated for 7 days (12\%) versus those treated for only 5 days (2.1\%) [21]. Another study reported lower incidence of rash in the 5-day cohort $(0.4 \%)$ versus the 7 -day cohort $(2.8 \%)$, overall in $1.6 \%$ of patients $[22]$. They also reported $3.1 \%$ of diarrhea as the adverse events of gemifloxacin [22]. Quinolones are associated with a nonspecific capacity to cause mast cells and basophils to release histamine, on the basis of animal studies measuring plasma histamine levels and the appearance of a generalized rash [23]. This may be, at least in part, a potential mechanism for some immune-mediated adverse reactions such as rash.

All the detected ADRs were evaluated according to Naranjo's algorithm probability scale; most of the ADRs were categorized as probable and as definite in only one patient. Majority of the ADRs were preventable in nature. In this study, we did not asses the types of ADRs. On severity scale, majority of these ADRs were moderate, followed by mild and in one patient the reaction was severe which appeared on the fixed dose combination therapy of amoxicillin-clavulanic acid (Augmentin) at the dose of 250:50 mg, four times daily frequency and on intramuscular administration. The most common organ systems affected with ADRs in our study were gastrointestinal and skin. This is supported by previous studies which reported gastrointestinal ADRs among the top three ADRs [17].

Antimicrobials have played important role in the treatment and cure of diseases; however, irrational prescribing, different types of races availability, variation in climatic conditions in Indian region may lead to differing types of ADRs in Indian population and these ADRs not only may result in hospital admission or prolonged hospitalization, increase economic burden but also may lead to permanent disability or even death. Awareness of the risk groups may decrease the cost as well as the incidence of preventable and serous antimicrobial related adverse effects. The most common antimicrobials involved in ADRs were newer one such as teicoplanin and gemifloxacin, although the numbers of prescription of these antimicrobials were one and two, respectively. As expected patients with more than two co-morbid con- ditions experienced higher percentage of ADRs. These findings need to be verified in larger number prescriptions containing these antimicrobials.

\section{KEY MESSAGE}

1. The association of generalized tonic clonic seizure with amoxicillin-clavulanic acid adverse drug reactions (ADRs) is particularly significant may be due to presence of $\beta$-lactam ring in $\beta$-lactam antibiotics.

2. A higher proportion of preventable ADRs were observed which can be prevented by careful monitoring of patient drug therapy.

\section{Conflict of interest}

No potential conflict of interest relevant to this article was reported.

\section{Acknowledgments}

The support by the University Grants Commission, New Delhi (Grant No. F. 4-1/2006 [BSR]/11-26/2008[BSR]) is gratefully acknowledged by the authors.

\section{REFERENCES}

1. Bone RC, Balk RA, Cerra FB, et al. Definitions for sepsis and organ failure and guidelines for the use of innovative therapies in sepsis. The ACCP/SCCM Consensus Conference Committee. American College of Chest Physicians/ Society of Critical Care Medicine. Chest 1992;101:16441655 .

2. Pierce G, Murray PR. Current controversies in the detection of septicemia. Eur J Clin Microbiol 1986;5:487-491.

3. Bone RC, Fisher CJ Jr, Clemmer TP, Slotman GJ, Metz CA, Balk RA. Sepsis syndrome: a valid clinical entity. Methylprednisolone Severe Sepsis Study Group. Crit Care Med 1989;17:389-393.

4. Meremikwu MM, Nwachukwu CE, Asuquo AE, Okebe JU, Utsalo SJ. Bacterial isolates from blood cultures of children with suspected septicaemia in Calabar, Nigeria. BMC Infect Dis 2005;5:110.

5. World Health Organization. Essential Newborn Care: Report of a Technical Working Group. Geneva: World 
Health Organization, 2005.

6. Fraser A, Paul M, Almanasreh N, et al. Benefit of appropriate empirical antibiotic treatment: thirty-day mortality and duration of hospital stay. Am J Med 2006;119:970-976.

7. International drug monitoring: the role of national centres. Report of a WHO meeting. World Health Organ Tech Rep Ser 1972;498:1-25.

8. Executive summary of disease management of drug hypersensitivity: a practice parameter. Joint Task Force on Practice Parameters, the American Academy of Allergy, Asthma and Immunology, the American Academy of Allergy, Asthma and Immunology, and the Joint Council of Allergy, Asthma and Immunology. Ann Allergy Asthma Immunol 1999;83(6 Pt 3):665-700.

9. Alam MS, Pillai PK, Kapur P, Pillai KK. Antimicrobial therapy and outcome of septicemia patients admitted to a university hospital in Delhi. Arzneimittelforschung 2012;62:117-122.

10. Munford RS. Severe sepsis and septic shock. In: Kasper DL, Braunwald E, Fauci AS, Hauser SL, Longo DL, Jameson JL, eds. Harrison's Principles of Internal Medicine. 16th ed. Vol. 2. New York: McGraw Hill, 2005:1606-1612.

11. Naranjo CA, Busto U, Sellers EM, et al. A method for estimating the probability of adverse drug reactions. Clin Pharmacol Ther 1981;30:239-245.

12. Hartwig SC, Siegel J, Schneider PJ. Preventability and severity assessment in reporting adverse drug reactions. Am J Hosp Pharm 1992;49:2229-2232.

13. Weiss J, Krebs S, Hoffmann C, et al. Survey of adverse drug reactions on a pediatric ward: a strategy for early and detailed detection. Pediatrics 2002;110(2 Pt 1):254-257.

14. Jha AK, Kuperman GJ, Teich JM, et al. Identifying adverse drug events: development of a computer-based monitor and comparison with chart review and stimulated volun- tary report. J Am Med Inform Assoc 1998;5:305-314.

15. Barranco P, Lopez-Serrano MC. General and epidemiological aspects of allergic drug reactions. Clin Exp Allergy 1998;28 Suppl 4:61-62.

16. Riedl MA, Casillas AM. Adverse drug reactions: types and treatment options. Am Fam Physician 2003;68:1781-1790.

17. Abdi SA, Churi S, Kumar YS. Study of drug utilization pattern of antihyperglycemic agents in a South Indian tertiary care teaching hospital. Indian J Pharmacol 2012;44:210-214.

18. Salvo F, Polimeni G, Moretti U, et al. Adverse drug reactions related to amoxicillin alone and in association with clavulanic acid: data from spontaneous reporting in Italy. J Antimicrob Chemother 2007;60:121-126.

19. GlaxoSmithKline. Product information: Augmentin XR (amoxicillin/clavulanate potassium) extended release tablets [Internet]. Durham (NC): GlaxoSmithKline, c20o6 [cited 2017 Aug 18]. Available from: https://www.accessdata.fda.gov/drugsatfda_docs/label/2008/050785soo7lbl.pdf.

20. Liang RH, Yung RW, Chan TK, et al. Ofloxacin versus co-trimoxazole for prevention of infection in neutropenic patients following cytotoxic chemotherapy. Antimicrob Agents Chemother 1990;34:215-218.

21. File TM Jr, Iannini PB. A profile of gemifloxacin, a new respiratory fluoroquinolone. Todays Ther Trends 2003;21:415-345.

22. File TM Jr, Mandell LA, Tillotson G, Kostov K, Georgiev O. Gemifloxacin once daily for 5 days versus 7 days for the treatment of community-acquired pneumonia: a randomized, multicentre, double-blind study. J Antimicrob Chemother 2007;60:112-120.

23. Kurata M, Kasuga Y, Nanba E, Nakamura H, Asano T, Haruta K. Flush induced by fluoroquinolones in canine skin. Inflamm Res 1995;44:461-465. 